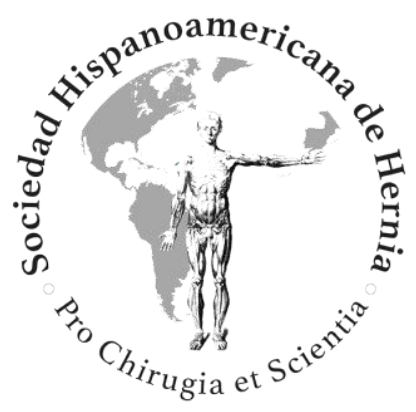

Hernia de Spiegel con apéndice cecal necrótico. Reporte de un caso

\title{
Spiegel hernia with necrotic cecal appendix. Case report
}




\section{Hernia de Spiegel con apéndice cecal necrótico. Reporte de un caso}

\section{Spiegel hernia with necrotic cecal appendix. Case report}

Gerardo Evaristo Méndez¹, Homero Plascencia Gálvez ${ }^{1}$, Enrique M. Covarrubias López ${ }^{2}$, Héctor U. González Romero', Víctor H. Grano González ${ }^{1}$

Servicios de ${ }^{1}$ Cirugía General y ${ }^{2}$ Anestesiología. Hospital Misión San Felipe. Zapopán, Jalisco (México)

Autor para correspondencia: Gerardo Evaristo Méndez. Servicio de Cirugía General. Hospital Misión San Felipe. C/ Virgen, 5586. Colonia Arboledas. 45070 Zapopán, Jalisco (México)

Correo electrónico: gevaristo5@yahoo.com.mx

Recibido: 02-03-2020

Aceptado: 09-03-2020

\section{RESUMEN}

Introducción: El diagnóstico de la hernia de Spiegel es difícil porque se presenta con manifestaciones clínicas vagas e inespecíficas. Además, suele desarrollarse a través de un orificio rígido por debajo de la aponeurosis del músculo oblicuo externo, lo que dificulta su detección por exploración física.

Objetivo: Nuestro objetivo es reportar un caso de apéndice cecal estrangulado y necrótico en una hernia de Spiegel.

Caso clínico: Varon de 47 años que presenta dolor abdominal de tres días de evolución. Su examen físico revela una masa irreducible de $10 \times$ $7 \mathrm{~cm}$ en el cuadrante inferior derecho del abdomen sin datos de irritación peritoneal. En la biometría hemática el recuento de leucocitos fue de $14.7 \times 10^{9} /$ L. La tomografía computarizada de abdomen 
describe una hernia de Spiegel con un cuello de $29 \mathrm{~mm}$ de diámetro conteniendo el apéndice cecal. En la cirugía de urgencia se encontra un apéndice cecal necrótico-hemorrágico dentro del saco herniario. La reparación de la hernia se reforzó con una malla de polipropileno entre el músculo oblicuo interno y la aponeurosis del oblicuo externo.

A siete meses del posoperatorio, el paciente se encuentra sin complicaciones de la herida quirúrgica.

Discusión: Un apéndice cecal necrótico en una hernia de Spiegel es una entidad muy rara reportada en la literatura médica. La colocación de material protésico en la reparación de las hernias de la pared abdominal no se recomienda en presencia de tejido estrangulado, pero puede ser una opción a criterio del cirujano.

\section{ABSTRACT}

Introduction: The diagnosis of Spiegel hernia is difficult because it presents with vague and nonspecific clinical manifestations. In addition, it usually develops through a rigid orifice below the aponeurosis of the external oblique muscle, which makes it difficult to detect by physical examination.

Objective: Our goal is to report a case of strangulated and necrotic cecal appendix in a Spiegel hernia.

Case report: A 47-year-old man presented abdominal pain of 3 days evolution. His physical examination revealed an irreducible mass of $10 \times$ $7 \mathrm{~cm}$ in the lower right quadrant of the abdomen with no evidence of peritoneal irritation. In blood count, the white blood cells were $14.7 \times$ 109 / L. Computed tomography of the abdomen described a Spiegel hernia with a neck $29 \mathrm{~mm}$ in diameter containing the cecal appendix. In emergency surgery, a necrotic-hemorrhagic cecal appendix was found inside the hernia sac. The hernia repair was reinforced with a polypropylene mesh between the internal oblique muscle and the 
external oblique aponeurosis. Seven months after the surgery, the patient was found without complications from the surgical wound.

Discussion: A necrotic cecal appendix in a Spiegel hernia is a very rare entity reported in the medical literature. The placement of prosthetic material in the repair of abdominal wall hernias is not recommended in the presence of strangulated tissue, but it may be an option at the surgeon's discretion.

Palabras clave: Hernia, Spiegel, apéndice.

Keywords: Hernia, Spiegel, appendix.

\section{INTRODUCCIÓN}

La hernia de Spiegel (HS) es la protrusión de un saco peritoneal, un órgano o grasa preperitoneal a través de un defecto, congénito o adquirido, de la aponeurosis del músculo transverso del abdomen entre la línea semilunar lateralmente y el músculo recto medialmente ${ }^{1}$. Su incidencia es de 1-2 \% de todas las hernias abdominales y tiene un ligero predominio de presentación en mujeres y entre la cuarta y la séptima década de vida².

La frecuencia de incarceración intestinal o de otro órgano en una HS es del 17-21 \% y la de estrangulación, de un $14 \%$ debido a que, en la mayoría de los casos, su cuello es $<2 \mathrm{~cm}$ de diámetro, por lo que casi en el $10 \%$ de los pacientes se requiere de una intervención quirúrgica urgente para la reparación de la hernia por estas complicaciones ${ }^{2,3}$.

El diagnóstico de la HS es difícil porque se presenta con manifestaciones clínicas vagas e inespecíficas en el historial médico de los pacientes. Además, suele desarrollarse a través de un orificio rígido y fibroso en los músculos transverso y oblicuo interno del abdomen sin atravesar, por lo general, la aponeurosis del oblicuo externo, lo que resulta en un número 
significativo de pacientes que presenta una hernia clínicamente difícil de detectar en la exploración física².

Nuestro objetivo es reportar un caso extremadamente raro de un apéndice cecal estrangulado y necrótico en una hernia de Spiegel.

\section{CASO CLÍNICO}

El 17 de julio de 2019 un hombre de 47 años de edad acudió al servicio de urgencias de nuestro hospital por dolor abdominal de tres días de evolución. Su historia médica no mostró comorbilidad. En el examen físico el paciente presentó signos vitales normales y una masa hiperémica, dolorosa espontáneamente y a la palpación, irreducible, con bordes mal definidos y un tamaño aproximado de $10 \times 7 \mathrm{~cm}$ localizada en el cuadrante inferior derecho del abdomen. No hubo datos de irritación peritoneal. Los estudios de laboratorio consistentes en una biometría hemática completa, química sanguínea, electrolitos séricos y tiempos de coagulación solo mostraron anormalmente un recuento de glóbulos blancos de $14.7 \times 10^{9} / \mathrm{L}$ (rango normal 4.5-10.0 × 109 / L). El reporte de la tomografía computarizada (TAC) de abdomen y pelvis describió una HS infraumblical derecha con un cuello de $29 \mathrm{~mm}$ de diámetro y un saco herniario de $10 \times 4 \mathrm{~cm}$ que contenía el apéndice cecal (fig. 1).

El paciente fue sometido a cirugía de urgencia bajo el diagnóstico de una HS complicada. Se le administró $1 \mathrm{~g}$ de ceftriaxona intravenosa una hora antes de la intervención quirúrgica. Se realizó una incisión transversa sobre el sitio de la masa hasta exponer y separar en la dirección de sus fibras el músculo oblicuo externo. Por un defecto de la aponeurosis spigeliana, entre el borde lateral del músculo recto del abdomen medialmente y la línea semilunar lateralmente protruía un saco incarcerado de consistencia dura (fig. 2). Se realizó una incisión de $1 \mathrm{~cm}$ medial al anillo de constricción para la liberación del saco, que se 
abrió y donde se encontró un apéndice cecal necrótico-hemorrágico y escaso líquido sanguinolento (fig. 3).

Se efectuó resección del apéndice ligando su base con material absorbible 2-0 y también se resecó el saco peritoneal. Se cerró el peritoneo con sutura continua absorbible 3-0 y posteriormente se irrigaron los tejidos con solución antiséptica de superoxidación, dejándola actuar durante 60 segundos. Se cerró el defecto herniario a través de los componentes músculo-aponeuróticos del transverso del abdomen y oblicuo interno con sutura interrumpida de polipropileno 2-0 y se repitió el procedimiento de irrigación con la solución antiséptica. La reparación de la hernia se reforzó con una malla de polipropileno colocada entre el músculo oblicuo interno y la aponeurosis del oblicuo externo, sobrepasando el anillo herniario $6 \mathrm{~cm}$ en todas direcciones (fig. 4). Su fijación y la aproximación de la aponeurosis del oblicuo externo se realizaron con sutura interrumpida de polipropileno 2-0. El tejido graso subcutáneo se cerró con sutura interrumpida absorbible 2-0 y la piel, con poliamida 3-0. Finalmente, se realizó infiltración local incisional con $20 \mathrm{ml}$ de ropivacaína $(7.5 \mathrm{mg} / \mathrm{mL}$ ).

El resultado histopatológico fue de un apéndice cecal necróticohemorrágico. La recuperación posoperatoria inmediata del paciente fue satisfactoria y dejó el hospital a los dos días de su intervención quirúrgica bajo tratamiento antibiótico vía oral con cefalexina (500 mg cada 8 horas) y metronidazol (500 mg cada 8 horas) durante cinco días. En la evaluación de seguimiento a los siete meses, el paciente se encontró bien, sin complicaciones posquirúrgicas.

\section{DISCUSIÓN}

Aunque la HS puede ocurrir en cualquier sitio de la aponeurosis spigeliana, que se extiende desde el $8 .^{\circ}$ cartílago costal hasta el pubis, en el $90 \%$ de los casos se localiza en una zona paramediana y transversal de $6 \mathrm{~cm}$ de ancho entre la espina ilíaca antero superior y la 
cicatriz umbilical (habitualmente por debajo del arco de Douglas), donde la aponeurosis es más amplia ${ }^{2,4}$.

La inconsistencia del examen físico para revelar una HS debido a una mayor frecuencia del tipo intersticial (debajo del músculo oblicuo externo), así como a su presentación con síntomas vagos, inespecíficos e intermitentes, pone de manifiesto el problema inherente de estas hernias para que no sean reconocidas, reportadas o diagnosticadas de forma temprana, excepto cuando existe una complicación, como la incarceración o la estrangulación, para que las haga clínicamente evidentes o más fácil de sospechar.

Se desconoce la causa de la HS, pero su aparición se ha asociado a factores de riesgo como trastornos del colágeno, edad avanzada, obesidad, rápida pérdida de peso, enfermedad pulmonar obstructiva crónica, traumatismos, antecedentes quirúrgicos y enfermedades congénitas ${ }^{5}$, ninguno de los cuales estuvo presente en la historia médica del caso que presentamos.

La TAC abdominal y pélvica es un método diagnóstico más preciso y confiable que el ultrasonido para la identificación de una HS complicada (incarcerada o estrangulada) o no complicada, en especial si se utiliza medio de contraste oral para poder revelar el posible contenido intestinal en el saco herniario ${ }^{6}$.

La TAC también permite visualizar las diferentes capas músculoaponeuróticas que participan en la formación del defecto herniario, además de poder excluir cualquier otra patología de la pared abdominal - intraabdominal como causa de la presentación clínica de los pacientes. En nuestro caso, la TAC simple puso de manifiesto tanto a la HS como al apéndice cecal incarcerado, además de la localización y el tamaño del defecto herniario, lo que nos permitió escoger el abordaje quirúrgico más adecuado para su resolución.

El saco de la HS contiene habitualmente grasa preperitoneal y epiplón mayor, aunque se ha reportado la presencia de intestino delgado o 
grueso, estómago, vesícula biliar, divertículo de Meckel, ovarios, testículos, leiomioma uterino y vejiga urinaria que protruían a través del defecto herniario ${ }^{1}$.

Aunque hay varios reportes de un apéndice cecal dentro de la HS, en la mayoría de los casos este fue normal, con mesenterio inflamado o con apendicitis aguda no complicada. Hasta donde sabemos, solo se han publicado hasta hoy siete casos, como en el paciente que presentamos, con un apéndice isquémico-necrótico dentro del saco de una $\mathrm{HS}^{4,6-11}$.

Consideramos que en nuestro caso la causa más probable del apéndice necrótico dentro del saco de la hernia fue la estrangulación de su aporte sanguíneo por un anillo herniario muy pequeño (29 mm de diámetro). Este tipo de hernia no es susceptible de manejo conservador debido a su alta frecuencia de incarceración y posterior estrangulación. Así, la reparación quirúrgica por un método abierto convencional, laparoscópico o robótico deberá realizarse poco después de hacer el diagnóstico, aunque en caso de sospechar una hernia complicada se recomienda el abordaje abierto para una intervención de urgencia, reservando su reparación mediante herniorrafía (cierre primario con sutura) para situaciones en las que la contaminación o la infección están presentes ${ }^{3,12}$. Sin embargo, en nuestro paciente colocamos una malla de polipropileno lo más alejada de los planos profundos potencialmente contaminados (entre el músculo oblicuo interno y la aponeurosis del oblicuo externo), de acuerdo con el método de Celdran y cols. ${ }^{13}$, y solo después de la irrigación con una solución antiséptica de los diferentes planos tisulares. Además, otros autores han reportado que el uso de mallas no absorbibles en campos contaminados es un procedimiento seguro ${ }^{14-16}$.

Así, siete meses después de la intervención no se han presentado complicaciones infecciosas o de otra índole en el sitio de la herida quirúrgica de nuestro paciente. 
En conclusión, la HS es un defecto herniario poco frecuente de la pared abdominal anterior. La TAC de abdomen puede poner de manifiesto su presencia y sus posibles complicaciones para un abordaje quirúrgico más directo. La colocación de material protésico para su reparación, en general, no se recomienda en presencia de tejido estrangulado, pero puede ser una opción a criterio del cirujano. Una amplia variedad de vísceras abdominales pueden encontrarse incarceradas dentro del saco herniario, pero la presencia de un apéndice cecal necrótico es una entidad muy rara reportada en la literatura médica.

\section{BIBLIOGRAFÍA}

1. Skandalakis PN, Zoras O, Skandalakis JE, et al. Spigelian hernia: surgical anatomy, embryology, and technique of repair. Am Surg. 2006;72:42-8. DOI: 10.1177/000313480607200110

2. Larson DW, Farley DR. Spigelian hernias: repair and outcome for 81 patients. World J Surg. 2002;26:1277-81. DOI: 10.1007/s00268002-6605-0

3. Moreno-Egea A, Carrasco L, Girela E, et al. Open vs laparoscopic repair of spigelian hernia: a prospective randomized trial. Arch Surg. 2002:137:1266-8. DOI: 10.1001/archsurg.137.11.1266

4. Thomasset SC, Villatoro E, Wood S, et al. An unusual Spigelian hernia involving the appendix: a case report. Cases J. 2010;3:22. DOI: 10.1186/1757-1626-3-22

5. Montes IS, Deysine M. Spigelian and other uncommon hernia repairs. Surg Clin North Am. 2003;83:1235-53. DOI: 10.1016/S0039-6109(03)00130-0

6. Onal A, Sökmen S, Atila K. Spigelian hernia associated with strangulation of the small bowel and appendix. Hernia. 2003;7:156-7. DOI: 10.1007/s10029-003-0118-0

7. Ramírez-Ramírez MM, Villanueva-Sáenz E. Rare hernias with atypical content: Apropos of a Spigelian hernia with acute 
apendicitis. Rev Gastroenterol Mex. 2017;82:181-2. DOI: 10.1016/j.rgmxen.2017.02.004

8. Hensley BJ, Stassen NA. Ruptured appendicitis within a left sided spigelian hernia in a patient status post previous transverse rectus abdominis myocutaneous flap resulting in necrotizing fasciitis. Am Surg. 2011;77:E294-5. DOI: 10.1177/000313481107701216

9. Jezieniecki Fernández C, Herreros Rodríguez J, Juárez Martín M, et al. Abdomen agudo por hernia de Spiegel complicada, un infrecuente caso que requiere alto grado de sospecha clínica. Rev Hispanoam Hernia. 2019;7:118-20. DOI: 10.20960/rhh.00187

10. Reinke C, Resnick A. Incarcerated appendix in a Spigelian hernia J Surg Case Rep. 2010;2010:3.

11. Lin $\mathrm{PH}$, Koffron $\mathrm{AJ}$, Heilizer $\mathrm{TJ}$, et al. Right lower quadrant abdominal pain due to appendicitis and an incarcerated spigelian hernia. Am Surg. 2000;66:725-7.

12.Jamshidian M, Stanek S, Sferra J, et al. Robotic repair of symptomatic Spigelian hernias: a series of three cases and surgical technique review. J Robot Surg. 2018;12:557-60. DOI: 10.1007/s11701-017-0742-9

13. Celdran A, Senaris J, Manas J, Frieyro O. The open mesh repair of Spigelian hernia. Am J Surg. 2007;193:111-3. DOI: 10.1016/j.amjsurg.2006.04.016

14. Velimezis G, Vassos N, Kapogiannatos G, et al. Strangulation and necrosis of right hemicolon as an extremely rare complication of Spigelian hernia. Arch Med Sci. 2016;12:469-72. DOI: 10.5114/aoms.2016.59273

15. Geisler DJ, Reilly JC, Vauhan SG, et al. Safety and outcome of use of nonabsorbable mesh for repair of fascial defects in the presence of open bowel. Dis Colon Rectum. 2003;46:1118-23. DOI: 10.1007/s10350-004-7290-x 
16. Kelly ME, Behrman SW. The safety and efficacy of prosthetic hernia repair in clean-contaminated and contaminated wounds. Am Surg. 2002;68:524-8. 